\title{
Expression of basigin in the early phase of acute myocardial ischemia in rats
}

\author{
YUN LIU ${ }^{1,2}$, WEI-BO LIANG ${ }^{1}$, LU-SHUN ZHANG ${ }^{1}$, LIN-BO GAO $^{1}$, TIAN-YI CHEN ${ }^{1}$, \\ YAN-YUN WANG ${ }^{3}$ and LIN $Z$ ZHANG $^{1}$ \\ ${ }^{1}$ Department of Forensic Biology, West China School of Preclinical and Forensic Medicine, Sichuan University, Chengdu, \\ Sichuan 610041; ${ }^{2}$ Department of Forensic Medicine, North Shichuan Medical College, Nanchong, Sichuan 637000; \\ ${ }^{3}$ Department of Immunology, West China School of Preclinical and Forensic Medicine, Sichuan University, \\ Chengdu, Sichuan 610041, P.R. China
}

Received November 2, 2012; Accepted March 7, 2013

DOI: $10.3892 / \mathrm{mmr} .2013 .1387$

\begin{abstract}
Basigin may be involved in cardiovascular disease. In our previous study, suppression subtractive hybridization results indicated that basigin may be associated with the early phase of acute myocardial ischemia (AMI) within $1 \mathrm{~h}$. However, little is known regarding the expression of basigin in the early phase of AMI. The aim of the present study was to evaluate the temporal and spatial expression patterns of basigin mRNA and protein levels in AMI in rats. We constructed an AMI model in rats that received left anterior descending coronary artery ligation for $0,15,30,60,120$ or $240 \mathrm{~min}$. Real-time quantitative PCR and in situ hybridization (ISH) were conducted to reveal the basigin mRNA levels in the early ischemic myocardium (EIM) and non-ischemic myocardium (NIM). The expression levels of basigin protein were detected using western blot analysis and immunohistochemistry. The expression levels of basigin mRNA and protein significantly changed in the EIM as early as 30 min from ischemia, and the changes continued to be present throughout the ischemic period $(\mathrm{P}<0.05)$. The expression levels of basigin mRNA were significantly reduced, whilst those of the protein underwent a significant $\sim 2$-fold increase in the EIM. However, there were no significant differences in the basigin mRNA or protein expression levels from $0-240 \mathrm{~min}$ in the NIM $(\mathrm{P}>0.05)$. We failed to detect a signal for basigin mRNA in the myocardium by ISH. Our findings indicated that basigin may be involved in acute myocardial ischemia following continual ischemia for $>30$ min.
\end{abstract}

Correspondence to: Professor Lin Zhang, Department of Forensic Biology, West China School of Preclinical and Forensic Medicine, Sichuan University, 17 Renmin South Road (Section III), Chengdu, Sichuan 610041, P.R. China

E-mail: zhanglin@scu.edu.cn

Key words: basigin, acute myocardial ischemia, rats

\section{Introduction}

Acute myocardial infarction is a major cause of sudden cardiac death (SCD) in patients with coronary artery disease and ischemic heart disease (1-3). The quantity of ischemic myocardium and extent of infarct expansion are crucial factors determining the occurrence of SCD during experimental acute myocardial ischemia (AMI), which may lead to congestive heart failure, aneurysm formation and myocardial rupture. Traditionally, the early myocardial damage has been identified using routine hematoxylin and eosin $(\mathrm{H} \& \mathrm{E})$ staining, following the occurrence of myocardial ischemic injury for $\geq 6 \mathrm{~h}$ (4). Certain studies have shown that myocardial apoptosis, histochemical techniques (including tetrazolium salts, phosphotungstic acid hematoxylin, trichrome, periodic acid-Schiff and hematoxylin-basic fuchsin-picric acid) and immunohistochemical staining may also be used to identify markers of myocardial damage 2-4 h following the onset of ischemia (1,5-8). However, these methods are not suitable for assessing the early phase of acute myocardial damage, particularly in cases where the patient succumbed within $1 \mathrm{~h}$. Their sensitivity and specificity remain controversial, due to the fact that positive results may sometimes be related to agonal factors and/or post-mortem changes (4). It may currently be challenging for forensic pathologists to make a post-mortem diagnosis of AMI when the ischemic insult occurred within minutes to $1 \mathrm{~h}$, as it is commonly asymptomatic or unrecognized. We have previously demonstrated that basigin may be associated with AMI within $1 \mathrm{~h}$ in rats by suppression subtractive hybridization, which revealed differentially expressed genes (9).

Basigin is also known as extracellular matrix metalloproteinase inducer (EMMPRIN), tumor cell-derived collagenase stimulatory factor (TCSF) and CD147. It was first identified on the surface of tumor cells, where it stimulated adjacent fibroblasts and endothelial or tumor cells to produce matrix metalloproteinases (MMPs), and facilitated the invasion of cancer cells (10-12). Basigin is also involved in tissue remodeling in numerous physiological and pathological conditions. Previously, certain studies have demonstrated that basigin is expressed in cardiac myocytes, but not in cardiac microvascular endothelial cells or cardiac fibroblasts $(13,14)$, and basigin 
protein expression levels are significantly elevated in acute infarcted myocardium $(15,16)$, destabilizing atheroma (17), human heart failure myocardium (13) and the ventricle of ischemic cardiomyopathy (18). A limited number of studies had investigated the theory that basigin mRNA and protein were expressed in AMI within $1 \mathrm{~h}$. In the present study, we investigated the temporal and spatial expression patterns of basigin in the early phase of AMI during different ischemic periods, as basigin is a known differentially expressed gene potentially related to the early phase of AMI.

\section{Materials and methods}

Animal model and tissue preparation. Adult healthy male Sprague-Dawley rats (weight, 200-250 g) were provided by the West China Medical Animal Center of Sichuan University (Chengdu, China). Experiments were performed with the approval of the Sichuan University Animal Research Committee), and were in accordance with animal protection guidelines (Guide for the Care and Use of Laboratory Animals, 8th edition). The animal models were created as previously described in detail (19). In brief, thoracotomy was performed in rats in the surgery group; the left anterior descending (LAD) coronary artery was ligated with a 6-0 silk suture by piercing the pericardial membrane, and electrocardiograms (ECGs) were recorded to confirm the successful ligation. Cervical dislocation of all experimental rats was performed $0,15,30$, 60, 120 and $240 \mathrm{~min}$ following ligation of the LAD coronary artery ( $\mathrm{n}=6$ for each time interval), respectively. Early ischemic myocardium (EIM) was assessed by $1 \%$ Evans blue. Additionally, EIM and proximate non-ischemic myocardium (NIM) were collected from each rat. The sham-operated rats $(n=36)$ were treated similarly to the rats receiving surgery, except they did not receive LAD coronary artery ligation, and the corresponding myocardium with EIM was collected and regarded as sham operation myocardium (SOM). Subsequently, the samples were fixed in $4 \%$ freshly made paraformaldehyde and embedded in optimal cutting temperature (OCT) medium for in situ hybridization (ISH) and immunohistochemistry (IHC), or were stored in liquid nitrogen for real-time quantitative PCR and western blot analysis.

Real-time quantitative PCR. Total RNA was extracted using the RNAiso Plus Isolation kit according to the manufacturer's instructions (Takara Bio Inc., Otsu, Japan). cDNA was synthesized from $500 \mathrm{ng}$ total RNA using oligo dT primers and a PrimeScript $^{\mathrm{TM}}$ RT Enzyme mix (Takara Bio Inc.).

Real-time quantitative PCR was conducted using the SYBR Premix Ex Taq $^{\mathrm{TM}}$ II kit (Takara Bio Inc.) in the ABI 7500 Fast system (Applied Biosystems, Foster City, CA, USA). According to Genbank (AY 120888.1), oligonucleotide primers for basigin were designed as follows: forward: 5'-GGCACCATCGTAACCTCTGT-3' and reverse: 5'-CAGGCTCAGGAAGGAAGATG-3' (size, 211 bp). The primers for the internal control, $\beta$-actin, were as follows: forward: 5'-TCACCCACACTGTGCCCATCTATGA-3' and reverse: 5'-CATCGGAACCGCTCATTGCCGATAG-3' (size, $300 \mathrm{bp}$ ). Samples without a template and samples processed without the reverse transcriptase step served as blank and negative controls, respectively. PCR amplification of cDNA was performed in a $20 \mu \mathrm{l}$ reaction volume containing $10 \mu \mathrm{l}$ SYBR Premix Ex Taq (X2) with $0.4 \mu$ l each primer, $0.4 \mu 1$ ROX Reference Dye and $2 \mu 1$ template cDNA. Cycling parameters were $95^{\circ} \mathrm{C}$ for $10 \mathrm{sec}$, followed by annealing and extension during 40 cycles of $95^{\circ} \mathrm{C}$ for $5 \mathrm{sec}, 60^{\circ} \mathrm{C}$ for $20 \mathrm{sec}$ and $72^{\circ} \mathrm{C}$ for $34 \mathrm{sec}$. The standard curves for each gene were generated by serial dilution of a plasmid containing basigin and $\beta$-actin cDNA, respectively, in order to quantify the mRNA concentrations. We confirmed the melting curve for detecting the SYBR-Green-based objective amplicon, as SYBR-Green also detected double-stranded DNA including primer dimers, contaminating DNA and PCR products from misannealed primers. Contaminating DNA or primer dimers appeared as peaks separated from the desired amplicon peak.

Each cDNA sample was run in triplicate, and the amplification efficiencies of primer pairs were determined by serial dilutions of the input template. The comparative cycle threshold method was used to analyze the data by generating relative values of the quantity of target cDNA. All data were normalized to correspond with $\beta$-actin mRNA, and were expressed as basigin mRNA in ischemic and non-ischemic myocardium during different ischemic periods. The relative difference in the initial amount of each mRNA species (or cDNA) was determined by comparing the $\mathrm{CT}$ values. The ratios of basigin $/ \beta$-actin were calculated to adjust for any variations in the real-time quantitative PCR reaction.

ISH. The oligonucleotide primers used for basigin reverse transcription-PCR were as follows: forward: 5'-GCTGTCTGTTGATGGGCTCG-3' and reverse: 5'-GGCTTCCGCCTCTTCTCGTA-3' (size, 786 bp) for the rat myocardium basigin cDNA (GenBank; AY 120888.1). PCR amplification was performed with AmpliTaq Gold ${ }^{\circledR}$ DNA Polymerase (Applied Biosystems, Foster City, CA, USA), and the PCR products were directly inserted into a pEASY-T3 Cloning vector (Beijing TransGen Biotech Co., Ltd., China). Plasmids were isolated and analyzed by restriction mapping and DNA sequencing. Digoxin (DIG)-labeled antisense and sense complementary RNA probes were prepared using DIG-labeled RNA kits (Sp6/T7; Roche Diagnostics GmbH, Mannheim, Germany), and plasmid cDNA was cloned as described in previous studies $(20,21)$. The hearts of two rats were evaluated by ISH per each ischemic time point, and at least six sections per heart were analyzed with the antisense basigin probe, including ischemic and non-ischemic myocardium, respectively $(\geq 18$ tissue sections were analyzed per time point). Briefly, frozen sections $(12 \mathrm{~mm})$ were fixed in $4 \%$ paraformaldehyde and phosphate-buffered saline (PBS) solution. The sections were then acetylated and dehydrated in preparation for hybridization with 2-40 $\mu \mathrm{g} / \mathrm{ml}$ RNase-free proteinase $\mathrm{K}$, for 10-30 min, and with/without $0.2 \mathrm{M} \mathrm{HCl}$ for $10 \mathrm{~min}$ at room temperature (RT), respectively. Quantification and efficiency of probe labeling were analyzed by serial dilution of test strips. Probes were used at a concentration of $2 \mu \mathrm{g} / \mathrm{ml}$. The slides were hybridized overnight with DIG-labeled antisense rat basigin probe per slide in a humidified chamber at $65^{\circ} \mathrm{C}$, and unbound probe was removed by RNase treatment and stringent washes. Nitro blue tetrazolium and 5-bromo-4-chloro-3-indolylphosphate were used for color detection. Tissues were examined with a Nikon phase contrast microscope (Nikon, Melville, NY, USA). Negative 

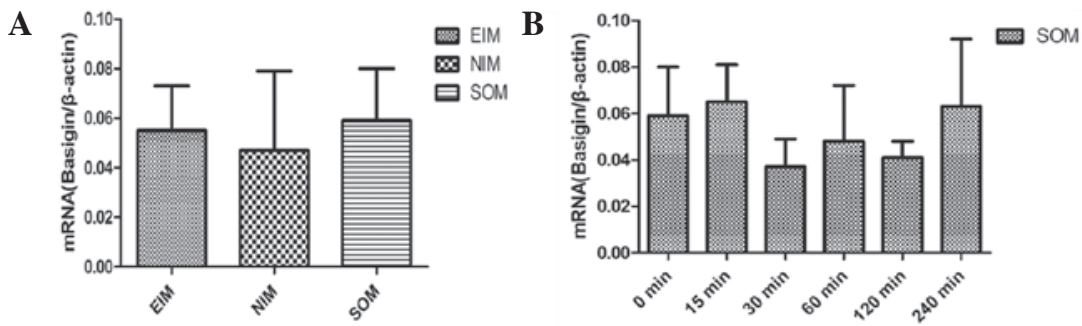

C

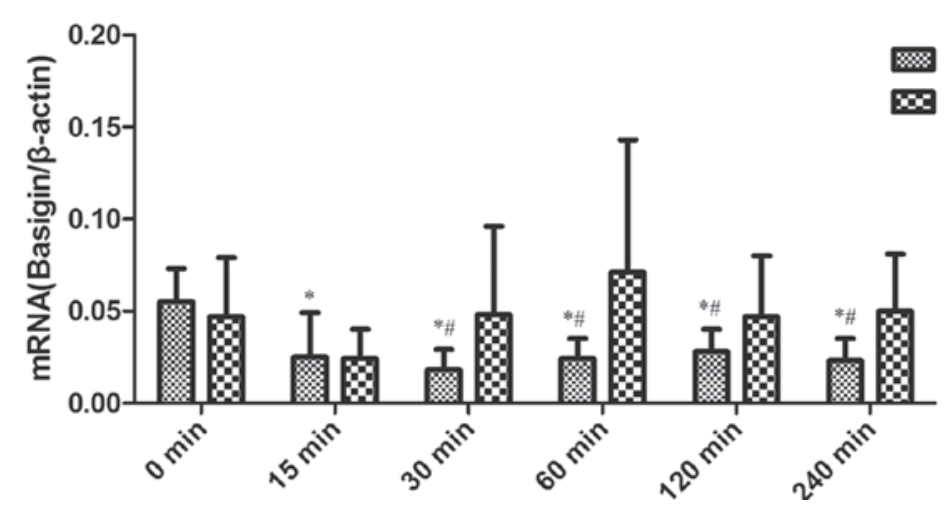

Figure 1. Basigin mRNA expression in the early ischemic myocardium (EIM) and non-ischemic myocardium (NIM) by real-time quantitative PCR. $\beta$-actin was used to confirm equal loading. (A) Basigin mRNA expression levels in the EIM, NIM and sham operation myocardium (SOM) were not significantly different at $0 \mathrm{~min}$. (B) Basigin mRNA expression levels in the SOM at different ischemic time points were not significantly different. (C) The expression of basigin mRNA in the EIM is lower than that in the NIM. "P<0.001 vs. the EIM at $0 \mathrm{~min}$; ${ }^{\prime} \mathrm{P}<0.05$ vs. the corresponding NIM.

and positive controls were conducted with sense basigin RNA probe and 4-day-old rat brain, respectively.

Western blot analysis. Basigin protein levels in AMI were analyzed by sodium dodecyl sulfate-polyacrylamide gel electrophoresis (SDS-PAGE). The tissue specimens were lysed in radioimmunoprecipitation assay (RIPA) buffer (50 mM Tris, pH 8.0; 150 mM NaCl; $1 \%$ Triton X-100; 1 mM EDTA, pH 8.0; and $0.1 \%$ SDS), supplemented with protease inhibitor cocktail $(50 \mu \mathrm{M})$, lactacystin $(20 \mu \mathrm{M}), \beta$-glycerophosphate $(25 \mathrm{mM})$ and sodium orthovanadate $(1 \mathrm{mM})$. Tissue proteins $(20 \mu \mathrm{g} / \mathrm{lane})$ were analyzed by continuous $10 \%$ SDS-PAGE, and the electrophoresed proteins were blotted onto a polyvinylidene difluoride (PVDF) membrane (BioRad, Hercules, CA, USA).

In order to reveal the basigin antigen, the membrane was blocked with $5 \%$ bovine serum albumin (BSA) in 5\% Tween-20 balanced salt Tris solution (TBST) for $1 \mathrm{~h}$ at RT, and subsequently incubated with rabbit anti-rat basigin antibody ( $1 \mathrm{mg} / \mathrm{ml}$, Abcam, Cambridge, MA, USA) overnight at $4^{\circ} \mathrm{C}$. Following primary antibody incubation, the membrane was washed with TBST and incubated for $1 \mathrm{~h}$ with secondary goat anti-rabbit IgG conjugated with horseradish peroxidase (Beijing Zhongshan Golden Bridge Biotechnology Co., Ltd., China). Finally, the signal was visualized with an enhanced chemiluminescence (ECL) kit (Amersham Biosciences Corp., Piscataway, NJ, USA) according to the manufacturer's instructions. The relative quantity of basigin was determined based on the signal intensity of the bands normalized to the $\beta$-actin signal in the same lane.

$I H C$. The frozen myocardial sections $(12 \mathrm{~mm})$ were fixed in $4 \%$ paraformaldehyde solution and blocked with $5 \%$ BSA prior to incubation at $4^{\circ} \mathrm{C}$ overnight with mouse anti-rat basigin (1:150; BD Biosciences, Franklin Lakes, NJ, USA). Bound antibody was labeled with goat anti-mouse secondary antibody conjugated with horseradish peroxidase $(1: 1000 ; 30 \mathrm{~min}$ at RT; Beijing Zhongshan Golden Bridge Biotechnology Co., Ltd.), and detected using a 3,3'-diaminobenzidine (DAB) kit and light microscopy. Irrelevant rabbit $\mathrm{IgG}$ was used for the primary layer as a negative control. The intensity of staining was semi-quantitatively assigned a score from 0-3, with the two observers blind to the source of the biopsy.

Statistical analysis. Data were presented as the mean \pm standard deviation (SD). The EIM was compared to the NIM for each group of rats by a paired Student's t-test. An unpaired $\mathrm{t}$-test and an analysis of variance (ANOVA) test were utilized among the different ischemic time points. $\mathrm{P} \leq 0.05$ was considered to indicate a statistically significant difference.

\section{Results}

Initial findings. No significant differences were observed in the expression levels of basigin mRNA among the EIM, NIM and $\mathrm{SOM}$ at $0 \mathrm{~min}(\mathrm{P}>0.05$; Fig. $1 \mathrm{~A})$. In addition, no significant differences were identified in either the basigin mRNA or protein expression levels in the SOM between different time points, by real-time quantitative PCR and western blot analysis, respectively (Fig. 1B and Fig. 2A). It was demonstrated that the AMI model in rats achieved by ligating the LAD coronary artery was mature, and that the result was stable as well as reliable for further experiments.

Expression of basigin $m R N A$. Basigin mRNA expression levels significantly decreased in the EIM following ischemia for 15-240 $\mathrm{min}$ compared with that at $0 \mathrm{~min}$, respectively $(\mathrm{P}<0.001$; Fig. 1C). However, no significant difference was observed in the NIM from 0-240 min ( $>>0.05)$. Basigin 
A

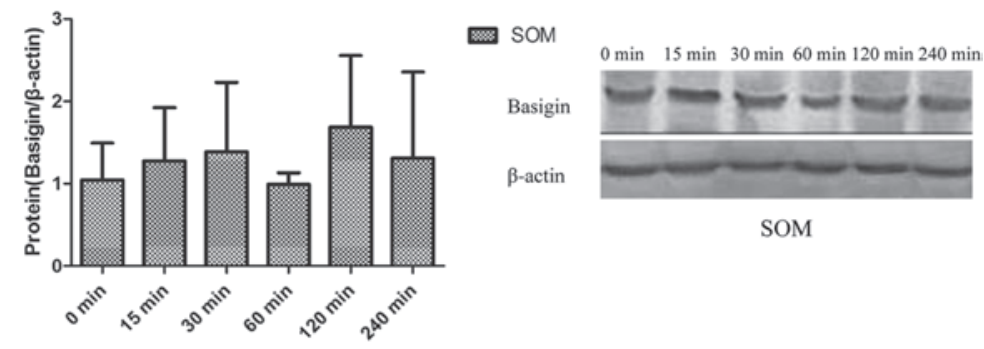

$\mathbf{B}$
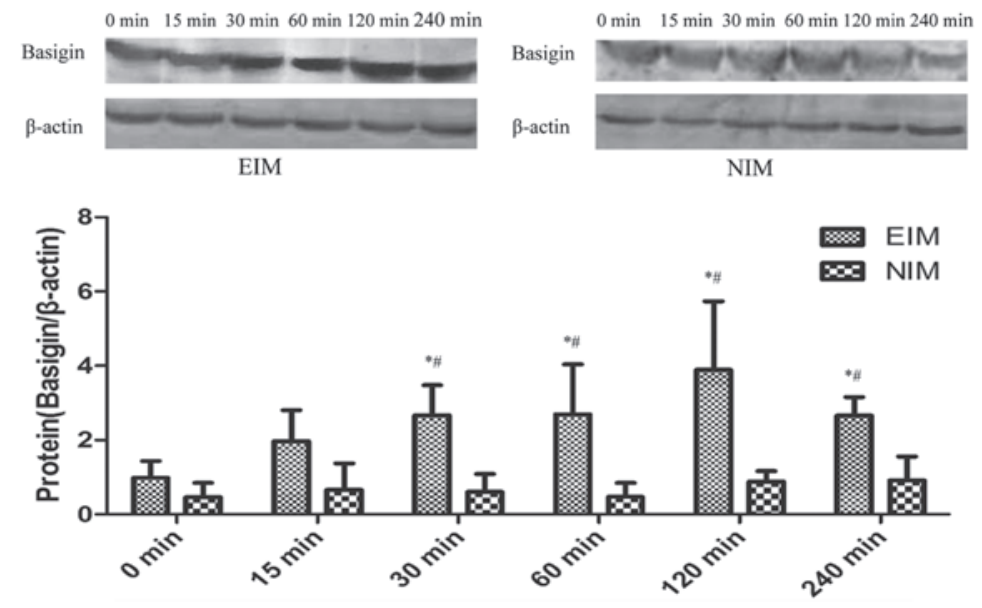

Figure 2. Basigin protein expression as detected in acute myocardial ischemia by western blot analysis. $\beta$-actin was used to confirm equal loading. (A) No significant difference was observed in basigin protein expression levels in the sham operation myocardium (SOM) between different ischemic time points (B) The expression of basigin protein in the early ischemic myocardium (EIM) was higher than that in the EIM at 0 min or in the corresponding non-ischemic myocardium (NIM). "P<0.05 vs. the EIM at 0 min; ${ }^{*} \mathrm{P}<0.05$ vs. the corresponding NIM.

mRNA expression levels decreased significantly in the EIM compared with that in the corresponding NIM following myocardial ischemia for $30 \mathrm{~min}(\mathrm{P}<0.05$; Fig. 1C).

We failed to detect an expression signal for basigin mRNA in the myocardium, including in the EIM, NIM and 4-day-old rat myocardium, using the DIG-labeled cRNA probe (Fig. 3A-C). However, normal neurons in the 4-day-old rat brain (the positive control) emitted a blue signal, which indicated the presence of basigin mRNA (Fig. 3D).

Basigin protein expression. Basigin was demonstrated to exist in the myocardium as a highly glycosylated (HG) form migrating at $58 \mathrm{kDa}$, as opposed to as a less-glycosylated (LG) form migrating at 32-44 kDa (Fig. 2A and B). The western blot analysis results revealed that basigin protein expression levels in the EIM following ischemia for 30-240 min significantly increased to approximately double that at $0 \mathrm{~min}$ in the EIM $(\mathrm{P}<0.05)$. We observed that basigin protein expression levels in the EIM were significantly different compared with those in the corresponding NIM following myocardial ischemia for 30 min $(\mathrm{P}<0.05$; Fig. 2B).

Basigin protein was observed to be mainly expressed on the membranes of normal cardiac myocytes, and occasionally expressed in the cytoplasm and nucleoli of cardiac myocytes by IHC. Positive basigin protein expression was found in the NIM and SOM at different ischemic periods, and in the EIM within 15 min following myocardial ischemia (Fig. 4A-D). Furthermore, its expression strongly increased in the EIM following myocardial ischemia for $>30 \mathrm{~min}$ (Fig. 4E-H). Additionally, the degree of positively stained cells was uniform in the EIM following myocardial ischemia for 30-240 min (Fig. 4E-H). The IHC results were concordant with the western blot analysis results.

\section{Discussion}

Basigin was initially characterized as a tumor cell surface glycoprotein, composed of two extracellular immunoglobulin domains, a transmembrane domain and a cytoplasmic domain $(10,22)$, which is distributed broadly in normal and tumor cells (23). Basigin has been most extensively investigated with respect to tumor invasion and metastasis, and it has been implicated as a factor contributing to the induction of MMP expression by autocrine or paracrine mechanisms (10,24-26). It has been demonstrated that basigin is expressed on cardiac myocytes $(13,14)$, and is involved with the human left ventricle, following acute myocardial infarction, heart failure myocardium and the ventricle of ischemic cardiomyopathies $(13,15,18)$. These results strongly suggested that basigin may be involved in myocardial injury.

The current study demonstrated that basigin mRNA and protein expression levels were significantly different from control levels as early as $30 \mathrm{~min}$ after the initiation of ischemia in the EIM, and the changes continued to be present throughout the ischemic period $(240 \mathrm{~min})$. The results were consistent with our previous study showing that basigin mRNA expression in EIM significantly decreased in the 15, 30 and 60 min groups compared with that of 0 min group (9), and certain signal pathway alterations of basigin in the ischemia/reperfusion myocardium, and irreversible myocardial cell injury 

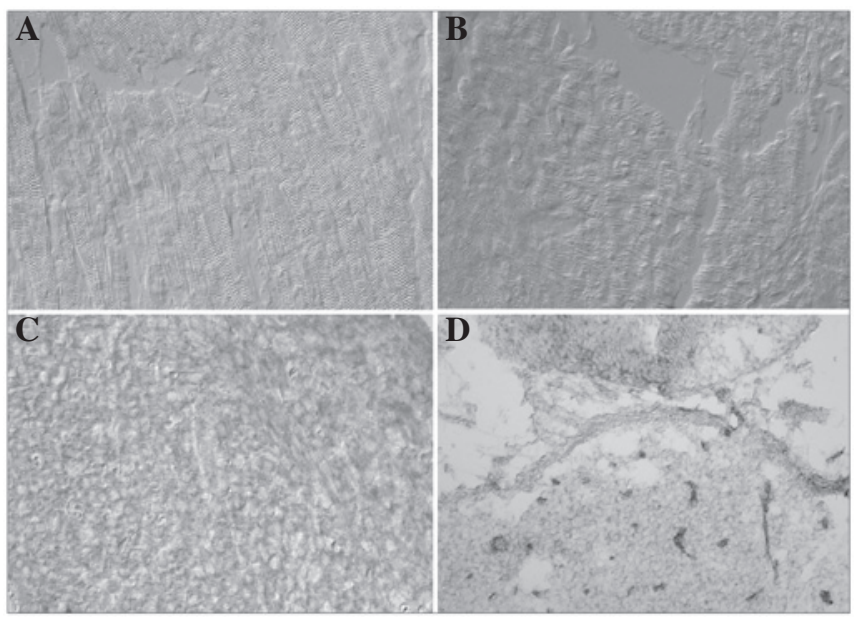

Figure 3. Localization of basigin mRNA in the myocardium and brain as detected by in situ hybridization. Basigin mRNA is not evident in adult (A) early ischemic myocardium (EIM); (B) non-ischemic myocardium (NIM); and (C) 4-day-old rat myocardium. (D) Normal neurons in the 4-day-old rat brain (the positive control), however, emit a blue signal that is indicative of basigin mRNA. Magnification, x200.
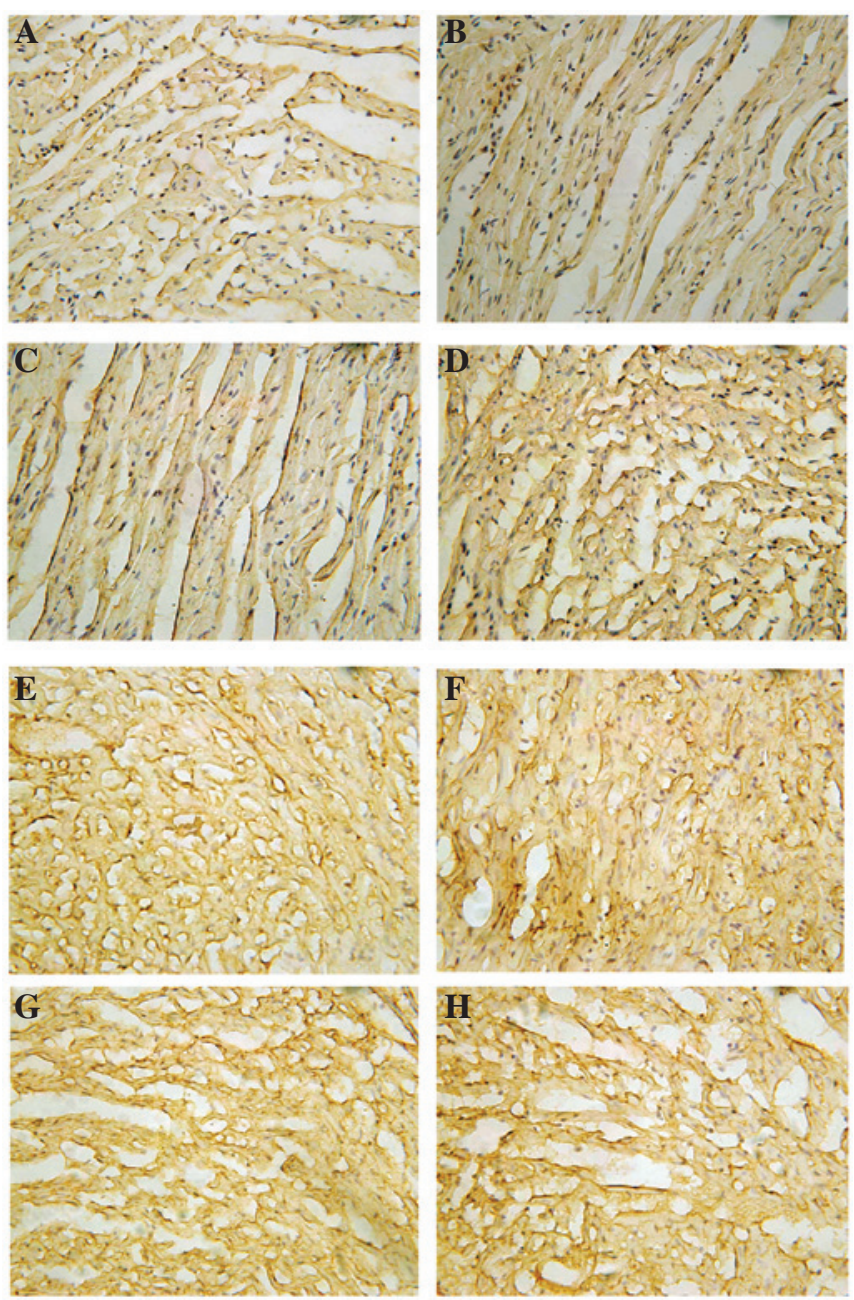

Figure 4. Basigin protein expression in acute myocardial ischemia as shown by immunohistochemistry. Positive basigin protein expression is evident in (A) the sham operation myocardium (SOM) group; (B) the non-ischemic myocardium (NIM) group; (C) the early ischemic myocardium (EIM) at $0 \mathrm{~min}$; and (D) the EIM with $15 \mathrm{~min}$ of ischemia. However, basigin protein expression strongly increased in the EIM with (E) 30, (F) 60, (G) 120 and (H) 240 min ischemia. Magnification, x200.
20-30 min following the occlusion of a coronary vessel (27). Similarly, phosphorylated MAPK family members, including ERK 1/2, SAPKs and p38 MAPK, demonstrated biphasic maximum levels at 5-10 min and 30 min through basigin (28). Foda et al demonstrated that basigin mRNA and protein expression levels increased in alveolar macrophages and airway epithelial cells within $1 \mathrm{~h}$ in ventilator-induced lung injury of rats (29). To the best of our knowledge, the present study is the first to demonstrate the accumulation feature of basigin at the early phase of AMI.

We demonstrated that basigin protein expression levels significantly increased in the EIM following ischemia for $>30 \mathrm{~min}$, and the protein was predominantly expressed on myocyte sarcolemma. This was concordant with other studies $(15,30-32)$ in which basigin expression significantly increased around the zone of necrosis and in monocytes in the acute myocardial infarction. Upregulation of monocarboxylate transporters (MCTs) and basigin expression in cardiac and neuronal cells under ischemic conditions has been observed (33-35); as well as the release of cyclophilin A (CyPA) from cardiac myocytes upon hypoxia/reoxygenation in vitro or ischemia/reperfusion in vivo, accompanied by the upregulation of basigin $(16,28)$. The correlation between basigin/CyPA/MCTs and related signaling may provide protection for cardiac myocytes and neurons under conditions of ischemic damage. However, basigin has the potential to induce extracellular matrix degradation by itself or by activating neighboring cells to induce certain MMPs [such as MMP-1, -2, -3 and -9, and membrane type 1(MT1)-MMP] in the myocardium, and by interactions with other proteins, which in turn may contribute to the progression of myocardial pathology (10,36-38). It has been suggested that basigin may be involved in the pathological processes during the early phase of AMI (ischemia >30 min). However, this issue has remained speculative and warrants further study.

Notably, we observed that the expression level of basigin mRNA was significantly reduced in the EIM. The correlation between basigin mRNA and protein expression was not consistent with certain studies $(29,39,40)$. For example, soluble basigin protein was significantly increased in proximal tubular epithelial cells by epidermal growth factor (EGF) and phorbol myristate acetate (PMA), although its mRNA level was not altered by these agents (40). In bleomycin-treated lungs, basigin mRNA was not present in areas of fibrosis, while coding protein levels were prominently increased in the fibro-inflammatory lesions (39). However, basigin mRNA and protein responses were correlated with increases in ventilator-induced lung injury of rats (29). Several theories have been proposed for the molecular basis of the differences of basigin mRNA and protein expression in the EIM. For example, Sp1 and Sp3 elements in the promoter of basigin may be critical for the regulation of basigin gene expression in macrophages, and a variety of stimuli affect their levels, activation and binding $(39,41)$. It has been demonstrated that the expression levels of basigin mRNA and protein are affected by different factors and so they may be involved in different pathological processes (39), or negative regulatory factors may be present (42). Another reason may be post-transcriptional mechanisms. We observed that only the HG form of basigin existed in the EIM, and not the LG form, indicating that 
basigin upregulation in the EIM may not be the cause of the ischemic myocardium. This finding was consistent with a study in which basigin potentially underwent significant translocation under certain circumstances (39). In addition, Joghetaei et al revealed that the migration of monocytes into aortic valve tissue may be affected by a basigin-based interaction of monocytes and endothelial cells (43).

In the present study, basigin mRNA levels were demonstrated to be significantly reduced in the EIM by real-time quantitative PCR; however, we were not able to detect an expression signal of basigin mRNA in either the EIM or the NIM by ISH. This was likely to be due to the low sensitivity of ISH compared with real-time quantitative PCR, and the basigin mRNA may have been of low abundance or low gene copy number in the myocardium.

In conclusion, the basigin gene may be involved in the myocardial pathophysiology process following continual myocardial ischemia for $>30 \mathrm{~min}$. We suggest that basigin may be identified as a predictor of acute myocardial ischemia in forensic medicine. Additionally, basigin may be a novel target for the clinical diagnosis and therapeutic intervention of acute myocardial ischemia.

\section{Acknowledgements}

This study was financially supported by a grant from the National Natural Science Foundation of China (grant no. 30973368/C1709).

\section{References}

1. Xu XH, Chen JG and Zhu JZ: Primary study of vascular endothelial growth factor immunohistochemical staining in the diagnosis of early acute myocardial ischemia. Forensic Sci Int 118: 11-14, 2001.

2. Ostadal B, Netuka I, Maly J, Besik J and Ostadalova I: Gender differences in cardiac ischemic injury and protection - experimental aspects. Exp Biol Med (Maywood) 234: 1011-1019, 2009.

3. Mehta D, Curwin J, Gomes JA and Fuster V: Sudden death in coronary artery disease: acute ischemia versus myocardial substrate. Circulation 96: 3215-3223, 1997.

4. Campobasso CP, Dell'Erba AS, Addante A, Zotti F, Marzullo A and Colonna MF: Sudden cardiac death and myocardial ischemia indicators: a comparative study of four immunohistochemical markers. Am J Forensic Med Pathol 29: 154-161, 2008.

5. Vargas SO, Sampson BA and Schoen FJ: Pathologic detection of early myocardial infarction: a critical review of the evolution and usefulness of modern techniques. Mod Pathol 12: 635-645, 1999.

6. Bardales RH, Hailey LS, Xie SS, Schaefer RF and Hsu SM: In situ apoptosis assay for the detection of early acute myocardial infarction. Am J Pathol 149: 821-829, 1996.

7. Xiaohong Z, Xiaorui C, Jun $\mathrm{H}$ and Qisheng Q: The contrast of immunohistochemical studies of myocardial fibrinogen and myoglobin in early myocardial ischemia in rats. Leg Med (Tokyo) 4: 47-51, 2002.

8. Lie JT, Holley KE, Kampa WR and Titus JL: New histochemical method for morphologic diagnosis of early stages of myocardial ischemia. Mayo Clin Proc 46: 319-327, 1971.

9. Liu Y, Gao LB, Liang WB, Pan XM, Wang YY, Xue H, Chen TY, Zhang LS, Zhu Y and Zhang L: The expression of Basigin mRNA in early ischemic myocardium and non-ischemic myocardium. Sichuan Da Xue Xue Bao Yi Xue Ban 41: 81-84, 2010 (In Chinese).

10. Biswas C, Zhang Y, DeCastro R, et al: The human tumor cell-derived collagenase stimulatory factor (renamed EMMPRIN) is a member of the immunoglobulin superfamily. Cancer Res 55: 434-439, 1995.

11. Biswas C: Tumor cell stimulation of collagenase production by fibroblasts. Biochem Biophys Res Commun 109: 1026-1034, 1982.
12. Caudroy S, Polette $\mathrm{M}$, Nawrocki-Raby $\mathrm{B}$, et al: EMMPRIN-mediated MMP regulation in tumor and endothelial cells. Clin Exp Metastasis 19: 697-702, 2002.

13. Spinale FG, Coker ML, Heung LJ, et al: A matrix metalloproteinase induction/activation system exists in the human left ventricular myocardium and is upregulated in heart failure. Circulation 102: 1944-1949, 2000.

14. Siwik DA, Kuster GM, Brahmbhatt JV, et al: EMMPRIN mediates beta-adrenergic receptor-stimulated matrix metalloproteinase activity in cardiac myocytes. J Mol Cell Cardiol 44: 210-217, 2008.

15. Nie R, Xie S, Du B, Liu X, Deng B and Wang J: Extracellular matrix metalloproteinase inducer (EMMPRIN) is increased in human left ventricle after acute myocardial infarction. Arch Med Res 40: 605-611, 2009.

16. Seizer P, Ochmann C, Schönberger T, et al: Disrupting the EMMPRIN (CD147)-cyclophilin A interaction reduces infarct size and preserves systolic function after myocardial ischemia and reperfusion. Arterioscler Thromb Vasc Biol 31: 1377-1386, 2011.

17. Yoon YW, Kwon HM, Hwang KC, et al: Upstream regulation of matrix metalloproteinase by EMMPRIN; extracellular matrix metalloproteinase inducer in advanced atherosclerotic plaque. Atherosclerosis 180: 37-44, 2005.

18. Spinale FG, Coker ML, Bond BR and Zellner JL: Myocardial matrix degradation and metalloproteinase activation in the failing heart: a potential therapeutic target. Cardiovasc Res 46: 225-238, 2000.

19. Zhang G, Zhou B, Zheng Y, et al: Time course proteomic profile of rat acute myocardial infarction by SELDI-TOF MS analysis. Int J Cardiol 131: 225-233, 2009.

20. Ushizawa K, Takahashi T, Hosoe M, et al: Gene expression profiles of novel caprine placental prolactin-related proteins similar to bovine placental prolactin-related proteins. BMC Dev Biol 7: 16, 2007.

21. Calmels TP and Mazurais D: In situ hybridization: a technique to study localization of cardiac gene expression. Methods Mol Biol 366: 159-180, 2007.

22. Guo H, Majmudar G, Jensen TC, Biswas C, Toole BP and Gordon MK: Characterization of the gene for human EMMPRIN, a tumor cell surface inducer of matrix metalloproteinases. Gene 220: 99-108, 1998.

23. Zheng HC, Wang W, Xu XY, et al: Up-regulated EMMPRIN/CD147 protein expression might play a role in colorectal carcinogenesis and its subsequent progression without an alteration of its glycosylation and mRNA level. J Cancer Res Clin Oncol 137: 585-596, 2011.

24. Suzuki S, Sato M, Senoo H and Ishikawa K: Direct cell-cell interaction enhances pro-MMP-2 production and activation in co-culture of laryngeal cancer cells and fibroblasts: involvement of EMMPRIN and MT1-MMP. Exp Cell Res 293: 259-266, 2004.

25. Hanata K, Yamaguchi N, Yoshikawa K, et al: Soluble EMMPRIN (extra-cellular matrix metalloproteinase inducer) stimulates the migration of HEp-2 human laryngeal carcinoma cells, accompanied by increased MMP-2 production in fibroblasts. Arch Histol Cytol 70: 267-277, 2007.

26. Kanekura T, Chen X and Kanzaki T: Basigin (CD147) is expressed on melanoma cells and induces tumor cell invasion by stimulating production of matrix metalloproteinases by fibroblasts. Int J Cancer 99: 520-528, 2002.

27. Sommers HM and Jennings RB: Experimental acute myocardial infarction; histologic and histochemical studies of early myocardial infarcts induced by temporary or permanent occlusion of a coronary artery. Lab Invest 13: 1491-1503, 1964.

28. Seko Y, Fujimura T, Taka H, Mineki R, Murayama K and Nagai R: Hypoxia followed by reoxygenation induces secretion of cyclophilin A from cultured rat cardiac myocytes. Biochem Biophys Res Commun 317: 162-168, 2004.

29. Foda HD, Rollo EE, Drews M, et al: Ventilator-induced lung injury upregulates and activates gelatinases and EMMPRIN: attenuation by the synthetic matrix metalloproteinase inhibitor, Prinomastat (AG3340). Am J Respir Cell Mol Biol 25: 717-724, 2001.

30. Schmidt R, Redecke V, Breitfeld Y, et al: EMMPRIN (CD 147) is a central activator of extracellular matrix degradation by Chlamydia pneumoniae-infected monocytes. Implications for plaque rupture. Thromb Haemost 95: 151-158, 2006.

31. May AE, Schmidt R, Bülbül BO, et al: Plasminogen and matrix metalloproteinase activation by enzymatically modified low density lipoproteins in monocytes and smooth muscle cells. Thromb Haemost 93: 710-715, 2005. 
32. Schmidt R, Bültmann A, Fischel S, et al: Extracellular matrix metalloproteinase inducer (CD147) is a novel receptor on platelets, activates platelets, and augments nuclear factor kappaB-dependent inflammation in monocytes. Circ Res 102: 302-309, 2008

33. Zhang F, Vannucci SJ, Philp NJ and Simpson IA: Monocarboxylate transporter expression in the spontaneous hypertensive rat: effect of stroke. J Neurosci Res 79: 139-145, 2005.

34. Han M, Trotta P, Coleman C and Linask KK: MCT-4, A511/Basigin and EF5 expression patterns during early chick cardiomyogenesis indicate cardiac cell differentiation occurs in a hypoxic environment. Dev Dyn 235: 124-131, 2006.

35. Kirk P, Wilson MC, Heddle C, Brown MH, Barclay AN and Halestrap AP: CD147 is tightly associated with lactate transporters MCT1 and MCT4 and facilitates their cell surface expression. EMBO J 19: 3896-3904, 2000.

36. Gabison EE, Mourah S, Steinfels E, et al: Differential expression of extracellular matrix metalloproteinase inducer (CD147) in normal and ulcerated corneas: role in epithelio-stromal interactions and matrix metalloproteinase induction. Am J Pathol 166: 209-219, 2005

37. Zavadzkas JA, Plyler RA, Bouges S, et al: Cardiac-restricted overexpression of extracellular matrix metalloproteinase inducer causes myocardial remodeling and dysfunction in aging mice. Am J Physiol Heart Circ Physiol 295: H1394-H1402, 2008.
38. Burggraf D, Liebetrau M, Martens HK, et al: Matrix metalloproteinase induction by EMMPRIN in experimental focal cerebral ischemia. Eur J Neurosci 22: 273-277, 2005.

39. Betsuyaku T, Kadomatsu K, Griffin GL, Muramatsu T and Senior RM: Increased basigin in bleomycin-induced lung injury. Am J Respir Cell Mol Biol 28: 600-606, 2003.

40. Shimada M, Yamabe H, Osawa H, et al: Extracellular matrix metalloproteinase inducer is expressed in the proximal tubular epithelial cells of the human kidney. Nephrology (Carlton) 14: 171-178, 2009.

41. Liang L, Major T and Bocan T: Characterization of the promoter of human extracellular matrix metalloproteinase inducer (EMMPRIN). Gene 282: 75-86, 2002.

42. Ruiz S, Castro-Castro A and Bustelo XR: CD147 inhibits the nuclear factor of activated T-cells by impairing Vav1 and Rac1 downstream signaling. J Biol Chem 283: 5554-5566, 2008.

43. Joghetaei N, Akhyari P, Rauch BH, et al: Extracellular matrix metalloproteinase inducer (CD147) and membrane type 1-matrix metalloproteinase are expressed on tissue macrophages in calcific aortic stenosis and induce transmigration in an artificial valve model. J Thorac Cardiovasc Surg 142: 191-198, 2010. 